\title{
High-Mobility Group Box-1 Protein in Adenine-Induced Chronic Renal Failure and the Influence of Gum Arabic Thereon
}

\author{
B. H. ALI ${ }^{1}$, M. AL ZA'ABI ${ }^{1}$, A. AL SHUKAILI ${ }^{2}$, A. NEMMAR ${ }^{3}$
}

${ }^{1}$ Department of Pharmacology and Clinical Pharmacy, College of Medicine and Health Sciences, Sultan Qaboos University, Oman, ${ }^{2}$ Department of Microbiology, College of Medicine and Health Sciences, Sultan Qaboos University, Oman, ${ }^{3}$ Department of Physiology, College of Medicine and Health Sciences, United Arab Emirates University, Al Ain, UAE

Received February 25, 2014

Accepted May 16, 2014

On-line September 5, 2014

\section{Summary}

Pathogenesis of adenine-induced chronic renal failure may involve inflammatory, immunological and/or oxidant mechanisms. Gum arabic (GA) is a complex polysaccharide that acts as an anti-oxidant which can modulate inflammatory and/or immunological processes. Therefore, we tested here the effect of GA treatment ( $15 \%$ in the drinking water for 4 weeks) in plasma and urine of rats, on a novel cytokine that has been shown to be pro-inflammatory, viz, DNA-binding high-mobility group box-1 protein (HMGB1). Adenine $(0.75 \%$ in the feed, 4 weeks) significantly increased indoxyl sulphate, urea and creatinine concentrations in plasma, and significantly decreased the creatinine clearance. GA significantly abated these effects. The concentrations of HMGB1 in urine before the start of the experiment were similar in all four groups. However, $24 \mathrm{~h}$ after the last treatment, adenine treatment increased significantly the concentration of HMGB1 when compared with the control. GA treatment did not affect the HMGB1 concentration in urine. Moreover, the concentration of HMGB1 in plasma obtained $24 \mathrm{~h}$ after the last treatment in rats treated with adenine was drastically reduced compared with the control group. This may explain its significant rise in urine. In conclusion, HMGB1 can be considered a potentially useful biomarker in adenine induced CRF and its treatment.

\section{Key words}

Chronic renal failure - Rats - Adenine - Gum Arabic • High-mobility group box-1 protein

\section{Corresponding author}

A. Nemmar, Department of Physiology, College of Medicine and Health Sciences, United Arab Emirates University, P.O. Box 17666, Al Ain, UAE. Fax: +971 3 7671966. E-mail: anemmar@uaeu.ac.ae, anemmar@hotmail.com

Adenine-induced chronic renal failure (CRF) is a rat model for human CRF that is increasingly being used, especially by Japanese workers, to study the pathophysiology of CRF and the agents that can be used to mitigate or prevent it (Ienaga et al. 2009). Adenineinduced CRF causes several biochemical, hormonal and molecular alterations that include increased circulatory fibroblast growth factor-23 (FGF-23) (Wang et al. 2013), and down regulation of the expression of the endothelial marker, CD31 and the upregulation of the expression of the mesenchymal markers, fibroblast specific-protein (FSP1) and $\alpha$-smooth muscle actin ( $\alpha$-SMA) (Wu et al. 2014).

Gum Arabic (GA [Acacia senegal $]$ ) is anecdotally reputed, in Arabian medicinal practices, to be useful in patients with chronic renal failure (CRF). In an attempt to find out more about the action of GA in CRF, we used this chemically induced-CRF model in rats to study the effect of treatment with GA on some biochemical, physiological, histoimmunochemical and histopathological actions produced in CRF (Ali et al. 2010) and on the actions produced by the CRF on certain behavioral actions in the rat (Ali et al. 2011), and 
confirmed the usefulness of GA in this model of CRF. The mechanism for the nephroprotection, however, remains uncertain. We have recently shown that a possible mechanism for the salutary effect of GA in adenine-induced CRF may be associated with amelioration of the adenine-induced inflammation and generation of free radicals (Ali et al. 2013).

In this work, we have looked at a novel inflammatory mediator, viz the DNA-binding highmobility group box-1 protein (HMGB1). This protein is thought to initiate and propagate inflammation when released by activated macrophages, and functions as a pro-inflammatory 'alarm cytokine' signaling tissue damage (Yang et al. 2005, Mantell et al. 2006, Oyama et al. 2010). Several recent studies reported the use of HMGB1 as a useful biomarker in various diseases including inflammation in retinal neuropathy induced by diabetes (El-Asrar et al. 2011, Abu El-Asrar et al. 2014). Some recent reports have shown an association between this protein and renal disease in humans (Bruchfeld et al. 2008, 2011). There is a single report in the literature suggesting elevated expression of HMGB1 in renal granulomatous inflammation nephritis induced by feeding adenine-rich diet to rats (Oyama et al. 2010). In this study, our data in rats show that adenine feeding $(0.75 \%$ for 4 weeks $)$ induced an increase of plasma indoxyl sulphate, urea and creatinine, and decreased creatinine clearance along with a significant increase in urinary concentration of HMGB1. While the pretreatment with GA prevented the increase of plasma urea and creatinine, and decreased creatinine clearance, it has not affected the HMGB1 concentration in urine. We also showed a decrease of HMGB1 in plasma in adenine-treated rats.

Male Wistar rats $(n=24)$, aged 8 weeks and initially weighing about $200 \mathrm{~g}$ were used. They were kept at a temperature of $22 \pm 2{ }^{\circ} \mathrm{C}$, relative humidity of $50 \%-60 \%$ and a 12:12-h light:dark cycle and were given a standard laboratory diet and water ad libitum. The rats were randomly divided into four equal groups and individually housed in metabolic cages, to facilitate urine collection 24-h before and 24-h after the last treatment. An acclimatization period of 6 days was allowed for the rats prior to experimentation. Rats were cared for under a protocol approved by the Animal Research Ethics Committee of our college and according to the NIH Guide for the Care and Use of Laboratory Animals, NIH publication no. 85-23, 1985.

Rats ( $\mathrm{n}=6$ in each group) were treated as follows: Group 1: Fed normal diet, and given normal saline (control, $0.25 \mathrm{ml} / \mathrm{rat}$ ), orally daily for 28 days. Group 2: Fed normal diet, and given GA (15\%) in the drinking water daily for 28 days. Group 3: Given adenine $(0.75 \% \mathrm{w} / \mathrm{w})$ in the feed, and given normal saline (control, $0.25 \mathrm{ml} / \mathrm{rat}$ ), orally daily for 28 days. Group 4: Given adenine $(0.75 \% \mathrm{w} / \mathrm{w})$ in the feed, and given GA $(15 \% \mathrm{w} / \mathrm{v})$ in the drinking water daily for 28 days.

The doses of adenine and GA used here were based on previous work (Mantell et al. 2006, Ali et al. 2010, 2011).

Twenty-four hours after the end of the treatment period, rats were anesthetized intraperitoneally with ketamine $(75 \mathrm{mg} / \mathrm{kg})$ and xylazine $(5 \mathrm{mg} / \mathrm{kg})$, and blood (4 ml) collected from the anterior vena cava and placed into heparinized tubes. The collected blood and urine were centrifuged at $900 \mathrm{~g}$ at $4{ }^{\circ} \mathrm{C}$ for $15 \mathrm{~min}$. The plasma obtained, together with the urine specimens were stored frozen at $-80{ }^{\circ} \mathrm{C}$ pending analysis.

To confirm the occurrence of CRF after adenine treatment, concentrations of indoxyl sulphate ( $\mathrm{Al}$ et al. 2013), creatinine and urea in plasma/urine were measured as described before (Mantell et al. 2006, Ali et al. 2010, 2011). The concentrations of HMGB1 in urine and plasma were measured using commercial ELISA kit bought from Shino-Test Corporation (Japan) according to the manufacturers' protocols. The detection limit of the kits was $0.2 \mathrm{ng} / \mathrm{ml}$. Concentrations below the limit were taken as zero in subsequent analyses.

Data are expressed as means \pm SEM. Comparisons between groups were performed by one way analysis of variance (ANOVA), followed by Newman-Keuls multiple range tests. $P$ values $<0.05$ were considered significant.

As shown in Table 1 , adenine feeding $(0.75 \%$ for 4 weeks) significantly decreased the body weight of rats and increased the urinary output and the plasma concentration of the urinary toxin indoxyl sulphate $(P<0.05)$. The treatment also caused significant increases $(P<0.05)$ in the concentrations of urea and creatinine in plasma, and significant decrease in the creatinine clearance $(P<0.01)$. Treatment with GA significantly abated the adenine effect (Table 1). 
Table 1. Effect of Gum Arabic (GA) on some physiological and biochemical parameters in plasma of rats treated with adenine $(0.75 \%$ w/w, 4 weeks).

\begin{tabular}{lcccc}
\hline \multicolumn{1}{c}{ Parameters/Group } & Control & Adenine & GA & Adenine + GA \\
\hline Urine output $(\mathrm{ml} / 24 \mathrm{~h})$ & $11.2 \pm 1.2$ & $31.3 \pm 3.2^{*}$ & $10.2 \pm 1.3$ & $18.8 \pm 2.3^{* *}$ \\
Change in body weight $(\%)$ & $22.3 \pm 2.2$ & $-40.2 \pm 6.0^{*}$ & $7.4 \pm 1.0$ & $-3.3 \pm 0.4^{* *}$ \\
Urea $(\mu \mathrm{mol} / \mathrm{l})$ & $6.3 \pm 0.8$ & $150.7 \pm 2.8^{*}$ & $13.9 \pm 1.2$ & $26.1 \pm 0.9^{* *}$ \\
Creatinine $(\mu \mathrm{mol} / \mathrm{l})$ & $63.0 \pm 4.7$ & $205.5 \pm 12.4^{*}$ & $54.5 \pm 3.0$ & $89.7 \pm 11.3^{* *}$ \\
Creatinine clearance $(\mathrm{ml} / \mathrm{min})$ & $1.0 \pm 0.2$ & $0.2 \pm 0.0^{*}$ & $0.9 \pm 0.1$ & $0.5 \pm 0.0^{* *}$ \\
Indoxyl sulfate $(\mu \mathrm{mol})$ & $1.7 \pm 0.8$ & $159.5 \pm 13.4^{*}$ & $0.0 \pm 0.0$ & $1.4 \pm 0.7^{* *}$ \\
\hline
\end{tabular}

Values in the table are mean $\pm \operatorname{SEM}(n=6)$. Adenine was added to the feed at a concentration of $0.75 \% \mathrm{w} / \mathrm{w}$, for 4 weeks, and GA (either alone or with adenine) was given in drinking water at $15 \% \mathrm{w} / \mathrm{v} .{ }^{*} P<0.05$ (Control vs all groups), ${ }^{* *} P<0.05$ (Adenine vs Adenine + gum).
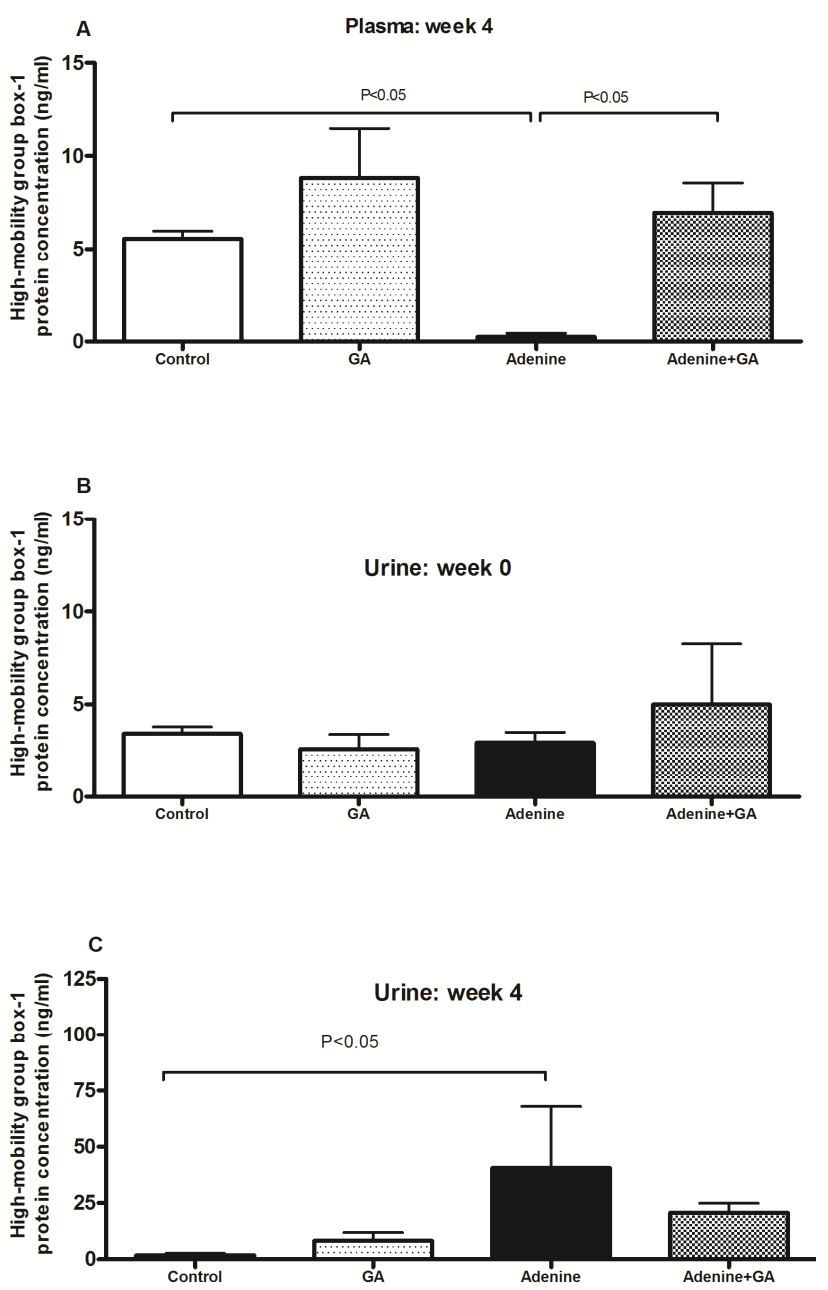

Fig. 1. High-mobility group box-1 concentration plasma (4 weeks, A) and urine (0, B and 4, C weeks) in control rats, and rats treated with adenine $(0.75 \% \mathrm{w} / \mathrm{w})$ in the feed, gum arabic (GA, $10 \% \mathrm{w} / \mathrm{v}$ in the drinking water) and with both adenine and GA given concomitantly at the same dose for 28 days. Each column and vertical bar represent mean \pm SEM. Differences between the groups were assessed by analysis of variance, followed by multiple comparison test. $P<0.05$ was considered significant.
Figure 1 depicts the effect of adenine-induced CRF and GA treatment on HMGB1 in urine before and after treatment and plasma 24-h after last treatment. The concentrations of HMGB1 in urine before the start of the experiment were similar in all four groups. However, 24-h after the last treatment, adenine treatment decreased significantly the concentration of HMGB1 in plasma and increased that in urine when compared with the controls. GA treatment decreased the HMGB1 concentration in the urine but this decrease did not reach statistical significance.

HMGB1 is an abundant and ubiquitous nuclear DNA-binding protein that had multiple functions dependent on its cellular location (Yang et al. 2005, Mantell et al. 2006, Oyama et al. 2010). In the nucleus, HMGB1 binds to DNA as a transcriptional regulator and accelerator of cellular motility on the cell surface. HMGB1 is released passively into the extracellular milieu by necrotic cells, but not by apoptotic cells, or is exported actively by monocytes/macrophages and neural cells upon receiving appropriate stimuli. In damaged tissue, extracellular HMGB1 acts as a necrotic signal, which alerts the surrounding cells and the immune system (Yang et al. 2005, Mantell et al. 2006, Bruchfeld et al. 2008, 2011, Oyama et al. 2010). Although extracellular HMGB1 can contribute to normal tissue development and repair, it is also implicated in the pathogenesis of many diseases (Arimura et al. 2009).

As reported before, in this work we confirmed that adenine feeding $(0.75 \%$ for 4 weeks $)$ caused significant decrease in body weight, and significant increases in the concentration of the uremic toxin indoxyl sulphate and that of urea and creatinine in plasma, and significant decrease in the creatinine clearance. Treatment 
with GA significantly abated the adenine effect (Table 1 and Fig. 1). Twenty-four hours after the last treatment, adenine treatment increased significantly the concentration of HMGB1 when compared with the controls. GA treatment did not affect the HMGB1 concentration in the urine. It has been reported that while HMGB1 was undetectable in urine of control rats, it appeared after 4 weeks, increased sharply after 12 weeks, and stayed at moderately increased level up to 20 weeks of $0.25 \%$ adenine fed rats (Oyama et al. 2010). Those authors also found that the level of HMGB1 increased with the dose of adenine, reaching a peak in rats fed $0.5 \%$ adenine at 5 weeks.

Our data show that the concentration of HMGB1 in plasma obtained $24 \mathrm{~h}$ after the last treatment in rats treated with adenine was drastically reduced compared with the other groups. This may explain its significant rise in urine. The other three groups had similar concentrations of HMGB1 in plasma. This result is not in line with the results obtained by Oyama et al. (2010) who reported an increase of serum HMGB1 occurring after 20 weeks in the $0.25 \%$ adenine-fed rats. The reasons for the disagreement is not known but could be related to the difference in the protocol used, rat strain or to the age of rats used. More work is needed to ascertain the reason for these discrepancies.

In conclusion, our data in rats show that adenine feeding $(0.75 \%$ for 4 weeks $)$ induced an increase of plasma indoxyl sulphate, urea and creatinine, and decreased creatinine clearance along with a significant increase in urinary concentration of HMGB1. While the pretreatment with GA prevented the increase of plasma urea and creatinine, and decreased creatinine clearance, it has not affected the HMGB1 concentration in urine. We also showed a decrease of HMGB1 in plasma in adeninetreated rats. Additional studies are needed to clarify the mechanisms underlying the role of HMGB1 in adenineinduced CRF. In a recent review, it has been reported that the measurement of HMGB1 in plasma may not reflect all its relevant activity in comparison with serum Western Blot (Yang et al. 2013). It would be of interest to conduct this work using this technique in future experiments.

\section{Conflict of Interest}

There is no conflict of interest.

\section{Acknowledgements}

This work was funded by the Research Council (TRC) in Oman (RC/MED/PHAR/10/01). Thanks are due to Ms. Sumiya Beegam for technical assistance.

\section{References}

ABU EL-ASRAR A, SIDDIQUEI M, NAWAZ M, GEBOES K, MOHAMMAD G: The proinflammatory cytokine high-mobility group box-1 mediates retinal neuropathy induced by diabetes. Mediators Inflamm 2014: 746415, 2014.

AL ZA'ABI M, ALI B, AL TM: HPLC-fluorescence method for measurement of the uremic toxin indoxyl sulfate in plasma. J Chromatogr Sci 51: 40-43, 2013.

ALI BH, AL SALAM S, AL HUSSENI I, KAYED RR, AL MASROORI N, AL HARTHI T, AL ZAABI M, NEMMAR A: Effects of Gum Arabic in rats with adenine-induced chronic renal failure. Exp Biol Med 235 : 373-382, 2010.

ALI BH, ZIADA A, AL HUSSEINI IS, BEEGAM S, NEMMAR A: Motor and behavioral changes in rats with adenine-induced chronic renal failure: Influence of acacia gum treatment. Exp Biol Med (Maywood) 236: 107-112, 2011.

ALI BH, AL-HUSSENI I, BEEGAM S, AL-SHUKAILI A, NEMMAR A, SCHIERLING S, QUEISSER N, SCHUPP $\mathrm{N}$ : Effect of gum arabic on oxidative stress and inflammation in adenine-induced chronic renal failure in rats. PLoS One 8: e55242, 2013.

ARIMURA N, KI-I Y, HASHIGUCHI T, KAWAHARA K, BISWAS KK, NAKAMURA M, SONODA Y, YAMAKIRI K, OKUBO A, SAKAMOTO T, MARUYAMA I: Intraocular expression and release of highmobility group box 1 protein in retinal detachment. Lab Invest 89: 278-289, 2009.

BRUCHFELD A, QURESHI AR, LINDHOLM B, BARANY P, YANG L, STENVINKEL P, TRACEY KJ: High Mobility Group Box Protein-1 correlates with renal function in chronic kidney disease (CKD). Mol Med 14: $109-115,2008$. 
BRUCHFELD A, WENDT M, BRATT J, QURESHI AR, CHAVAN S, TRACEY KJ, PALMBLAD K, GUNNARSSON I: High-mobility group box-1 protein (HMGB1) is increased in antineutrophilic cytoplasmatic antibody (ANCA)-associated vasculitis with renal manifestations. Mol Med 17: 29-35, 2011.

EL-ASRAR AM, NAWAZ MI, KANGAVE D, GEBOES K, OLA MS, AHMAD S, AL-SHABRAWEY M: High-mobility group box-1 and biomarkers of inflammation in the vitreous from patients with proliferative diabetic retinopathy. Mol Vis 17: 1829-1838, 2011.

IENAGA K, MIKAMI H, YOKOZAWA T: First indications demonstrating the preventive effects of NZ-419, a novel intrinsic antioxidant, on the initiation and/or progression of chronic renal failure in rats. Biol Pharm Bull 32: 1204-1208, 2009.

MANTELL LL, PARRISH WR, ULLOA L: HMGB-1 as a therapeutic target for infectious and inflammatory disorders. Shock 25: 4-11, 2006.

OYAMA Y, HASHIGUCHI T, TANIGUCHI N, TANCHAROEN S, UCHIMURA T, BISWAS KK, KAWAHARA K, NITANDA T, UMEKITA Y, LOTZ M, MARUYAMA I: High-mobility group box-1 protein promotes granulomatous nephritis in adenine-induced nephropathy. Lab Invest 90: 853-866, 2010.

WANG B, JHA JC, HAGIWARA S, MCCLELLAND AD, JANDELEIT-DAHM K, THOMAS MC, COOPER ME, KANTHARIDIS P: Transforming growth factor-beta1-mediated renal fibrosis is dependent on the regulation of transforming growth factor receptor 1 expression by let-7b. Kidney Int 85: 352-361, 2013.

WU M, TANG RN, LIU H, XU M, PAN MM, LIU BC: Cinacalcet attenuates the renal endothelial-to-mesenchymal transition in rats with adenine-induced renal failure. Am J Physiol Renal Physiol 306: F138-F146, 2014.

YANG H, WANG HC, CZURA CJ, TRACEY KJ: The cytokine activity of HMGB1. J Leukoc Biol 78: 1-8, 2005.

YANG H, ANTOINE DJ, ANDERSSON U, TRACEY KJ: The many faces of HMGB1: molecular structure-functional activity in inflammation, apoptosis, and chemotaxis. J Leukoc Biol 93: 865-873, 2013. 